\title{
The Baikal neutrino experiment: from NT200 to NT200+
}

\author{
V. Aynutdinov ${ }^{a}$, V.Balkanov ${ }^{a}$, I. Belolaptikov ${ }^{g}$, N.Budnev $^{b}$, L. Bezrukov ${ }^{a}$, D. Borschev $^{a}$, \\ A.Chensky $^{b}$, I. Danilchenko ${ }^{a}$, Ya.Davidov ${ }^{a}, Z_{\text {Zh.-A. Djilkibaev }}^{a}$, G. Domogatsky $^{a}$,

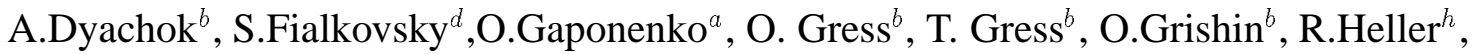 \\ A.Klabukov $^{a}$, A.Klimov ${ }^{f}$, K.Konischev ${ }^{g}$, A.Koshechkin ${ }^{a}$, L.Kuzmichev ${ }^{c}$, V.Kulepov ${ }^{d}$, \\ B.Lubsandorzhiev $^{a}$, S.Mikheyev ${ }^{a}$, M.Milenin $^{d}$, R.Mirgazov $^{b}$, T.Mikolajski $^{h}$, E.Osipova $^{c}$,

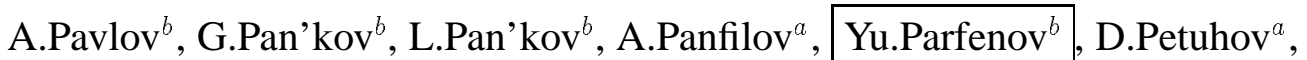 \\ E.Pliskovsky ${ }^{g}$, P.Pokhil $^{a}$, V.Polecshuk ${ }^{a}$, E.Popova ${ }^{c}$, V.Prosin $^{c}$, M.Rozanov $^{e}$, \\ V.Rubtzov $^{b}$, B.Shaibonov ${ }^{a}$, A.Shirokov ${ }^{c}$,Ch. Spiering ${ }^{h}$, B.Tarashansky ${ }^{b}$, \\ R.Vasiliev $^{g}$, E.Vyatchin $^{a}$, R.Wischnewski ${ }^{h}$, I.Yashin $^{c}$, V.Zhukov $^{a}$ \\ (a) Institute for Nuclear Reseach, Russia \\ (b) Irkutsk State University, Russia \\ (c) Skobeltsin Institute of Nuclear Physics, Moscow State University, Russia \\ (d) Nizni Novgorod State Technical University, Russia \\ (e) St.Petersburg State Marine Technical University, Russia \\ (f) Kurchatov Institute, Russia \\ (g) Joint Institute for Nuclear Research, Dubna, Russia \\ (h) DESY, Zeuthen, Germany \\ Presenter: Kuzmichev L.A. (kuz@dec1.sinp.msu.ru), rus-kuzmichev-1A-abs3-og25-oral
}

The Baikal neutrino telescope NT200 takes data since 1998. In 2005, the deployment of three additional strings for common operation with NT200 was finished. We describe the physics program and the design of the new telescope named NT200+ and present selected physical results obtained with NT200. First results from NT200+ will be presented at the conference.

\section{Introduction}

The Baikal Neutrino Telescope is operated in Lake Baikal, Siberia, at a depth of $1.1 \mathrm{~km}, 3.6 \mathrm{~km}$ from the shore. Deep Baikal water is characterized by an absorption length of $L_{a b s}(480 \mathrm{~nm})=20 \div 24 \mathrm{~m}$, a scattering length of $L_{s}-30 \div 70 \mathrm{~m}$ and a strongly anisotropic scattering function with a mean cosine of scattering angle $0.85 \div 0.9$. The present stage of telescope, NT200+ (fig II was put into operation at April 9th, 2005. The new configuration consists of the old NT200 telescope plus the three new, external strings, placed $100 \mathrm{~m}$ from the center of NT200. With the new strings, the sensitivity of Baikal telescope for very high energy neutrinos increases by a factor 4 . Below we describe the new telescope and present selected physical and methodical results. Results on the search for high energy neutrinos and relativistic monopoles are presented in separate papers.

\section{NT200+}

NT200 plus the new external strings form NT200+. NT200 | I with 192 optical modules(OM), was put into operation in 1998. An umbrella-like frame carries 8 strings $72 \mathrm{~m}$ long, each with 24 pairwise arranged OMs. The optical modules contain 37-cm photodetectors QUASAR-370 [2, which have been developed specially for our project. The OMs are grouped in pairs along the strings. The PMs of a pair are switched in coincidence in order to suppress background from bioluminescence and PM noise. A pair defines a channel. 


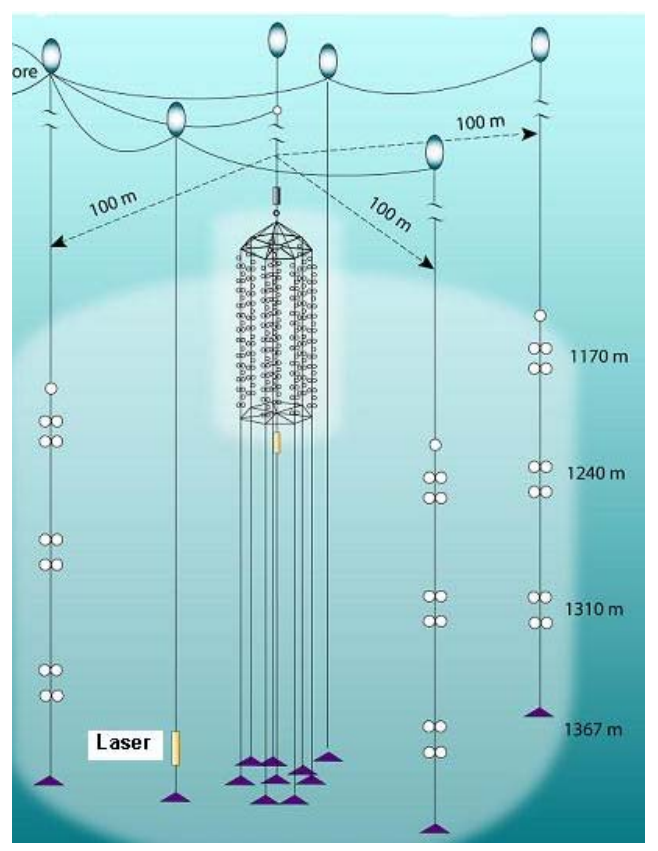

Figure 1. Sketch of NT200+

A trigger is formed by requiring $\geq N$ hits (with hit refering to a channel) within $500 \mathrm{~ns}$. $N$ is typically set to 3 or 4 . This signals is used as a common stop for TDCs. For the common operation with the external strings the signal of the trigger is sent through $1.2 \mathrm{~km}$ coaxial cable to the underwater DAQ center of NT200+ (20 $\mathrm{m}$ below surface, distributed over several glass spheres). For time calibration of NT200, two nitrogen lasers, placed on the top and below the central string, are used. The light pulse from the first laser $(\lambda=480 \mathrm{~nm})$ is sent to all OMs via optical fibers with equal length, the light pulse from the second is emitted straight to the water.

External strings $140 \mathrm{~m}$ long are placed at 100 meter distance from the center of NT200, each with 12 OMs grouped in pairs like in NT200. The upper pairs are at approximately the same level as the bottom OMs of NT200. On each external string, an independent string trigger is formed in case the number of fired channels is $\geq 2$ within 1000 ns. String triggers are sent to the underwater DAQ center, where the time difference between string trigger and the trigger of NT-200 is measured. This information is used to relate the times within an event of NT200 OMs and OMs of the externals strings. For time offset synchronization of the external strings with respect to NT200, an additional laser, placed on a separate string (see fig II left bottom) is used. This laser emits ten times more photons $\left(\geq 10^{12}\right)$ than the old ones and fires OMs on all externals strings as well as on NT200. With this laser it was found that the jitter of the time difference between OMs of NT200 and OMs of the external strings is smaller than $3 \mathrm{~ns}$.

Our search strategy for high energy neutrinos relies on the detection of the Cherenkov light emitted by cascades, produced by neutrino interactions in a large volume mostly below NT200. The detection volume for NT200+ is shown in fig $\boldsymbol{\nu}$ With three additional strings, it is increased by four times. With NT200+ we also significantly increase the accuracy of the position of the cascade vertex (fig 3 within the volume spanned by the outer strings, and based on that the energy reconstruction.

For the first 1000 hours of operation, $7.5 \cdot 10^{4}$ common events (detected by NT200 and external strings) have been collected. Data analysis is in progress and first results will be presented at the conference. 


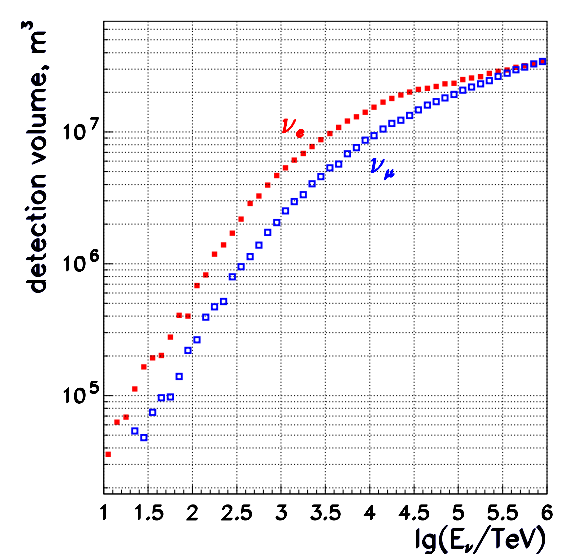

Figure 2. Detection volume of NT200+ for $\nu_{e}$ and $\nu_{\mu}$ events which survives all cuts

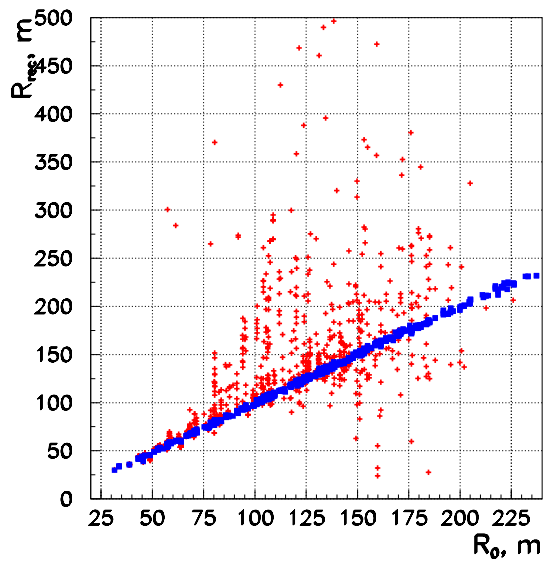

Figure 3. Reconstructed v. simulated coordinates of cascades in NT-200+ (rectangles) and NT-200(crosses)

\section{Selected results}

\subsection{Atmospheric Muon Neutrinos}

The signature of charged current muon neutrino events is a muon crossing the detector from below. Muon track reconstruction algorithms and background rejection have been described elsewhere 3 . Compared to 3 , the analysis of the 5-year sample (1038 days for pure operation time) was optimized for higher signal passing rate, and accepting a slightly higher contamination of $15-20 \%$ fake events. A total of 372 upgoing neutrino candidates were found. From Monte-Carlo simulations, a total 385 atmospheric neutrino and background events are expected. The skyplot of these events is shown in fig 4

\subsection{Search for Neutrinos from WIMP Annihilation}

The search for WIMPs with the Baikal neutrino telescope is based on a possible signal of nearly vertically upward going muons, exceeding the flux of atmospheric neutrinos. The applied cuts for event selection [ 5 result in a detection area of $1800 \mathrm{~m}^{2}$ for vertically upward going muons. In 502 days effective data taking, 24 events with $-0.75 \geq \cos (\theta) \geq-1$ have been found, in accordance with expectations for atmospheric neutrinos. Regarding these 24 events as being induced by atmospheric neutrinos, one can derive an upper limit on the excess flux of muons from the center of the Earth due to annihilation of neutralinos (fig. 5 .

\subsection{Search for acoustic signals at Lake Baikal}

In 2001-2003, a series of experiments has been performed to detect acoustic signals in water from EAS. For these experiments, a small EAS array was deployed on the ice. A special acoustic device recorded sound signals for a $100 \mathrm{~ms}$ interval after the EAS time. This device consists of 4 hydrophones arranged at the vertices of a pyramide with $1.5 \mathrm{~m}$ side length. During these experiments many bipolar signals from close to the EAS core were detected, but due the very high level of acoustic noise we cannot prove that these signals are really from EAS 4 . In 2005, four hydrophones with a new data acquisition system were tested in situ. With this device it is possible to reconstruct the direction to the sound source with one degree accuracy. During a 20hour test with the device at $100 \mathrm{~m}$ depth it was found that the majority of the acoustic signals comes from the surface of the lake and only a few from below the horizon. No signals from directions steeper than $20^{\circ}$ below horizon have been detected. In 2006 we plan to operate this device together with NT200+. 


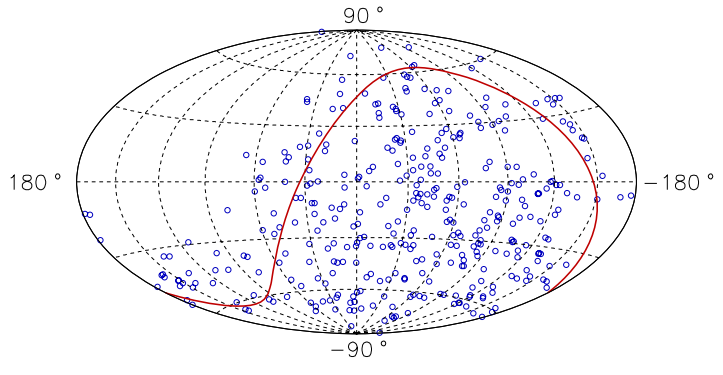

Figure 4. Skyplot of neutrino events in galactic coordinates for five years

\section{Conclusion and Outlook}

The Baikal Neutrino telescope NT200, operated from 1998, was upgraded to NT200+, a 10-Mton detector with a sensitivity of approximately $\Phi_{\nu_{e}} E^{2} \sim 10^{-7} \mathrm{~cm}^{-2} \mathrm{sr}^{-1} \mathrm{GeV}$ for diffuse neutrino fluxes at $E \geq 10^{2} \mathrm{TeV}$. NT200+ will search for neutrinos from AGNs, GRBs and other extraterrestrial sources, neutrinos from cosmic ray interactions in the Galaxy as well as high energy atmospheric muons with $E_{\mu} \geq 10 \mathrm{TeV}$,and also for exotic particles (monopoles, strangles, Q-balls). In parallel to this short term goal, we started research \& development activities towards a Gigaton Volume Detector 14 in Lake Baikal. Our last results on the search for high energy neutrinos and relativistic monopoles and more detailed descriptions of the data acquisition system of NT200+ are presented in separate papers on this conference 78 .

This work was supported by the Russian Ministry of Education and Science, the German Ministry of Education and Research and the Russian Fund of Basic Research (gratns 05-02-17476, 04-02-17289, 04-02-16171, 0502-16593), by the Grant of the President of Russia NSh-1828.2003.2, and by NATO-Grant NIG-981707

\section{References}

[1] I. Belolaptikov et al., Astropart.Phys. 7, 263 (1997).

[2] R. I. Bagduev et al., Nucl.Instr.Meth. A420, 138(1999).

[3] I. Belolaptikov et al., Astropart.Ph. 12, 75(1999)

[4] V. Balkanov et al., Nucl. Phys.B (Proc.Suppl.)118, 363(2003)

[5] V. Balkanov et al., Nucl.Phys.(Proc.Suppl.) 91, 438(2000)

[6] C. Spiering. astro-ph/0503122 (2005)

[7] V. Aynutdinov et al.[Baikal Collaboration], these proceedings: ger-wischnewski-R-abs1-og25-oral

[8] V. Aynutdinov et al.[Baikal Collaboration], these proceedings: ger-wischnewski-R-abs1-he23-poster 